\title{
Regulation of retinol binding protein 4 production in primary human adipocytes by adiponectin, troglitazone and TNF- $\alpha$
}

\author{
H. Sell • J. Eckel
}

Received: 7 May 2007 / Accepted: 18 June 2007 / Published online: 28 July 2007

(C) Springer-Verlag 2007

Keywords Adipokine Human adipocytes ·

Insulin resistance $\cdot$ Obesity $\cdot \mathrm{RBP} 4$

\begin{abstract}
Abbreviations
ANP atrial natriuretic peptide

PPAR $\gamma$ peroxisome proliferator-activated receptor $\gamma$

RBP4 retinol binding protein 4
\end{abstract}

To the Editor: Adipose tissue has an important endocrine function, producing a variety of bioactive proteins that may regulate energy metabolism and insulin sensitivity. It releases a variety of the so-called adipokines, including retinol binding protein 4 (RBP4), which has recently been described as a link between obesity and insulin resistance [1] in addition to its primary function as a specific carrier of vitamin $\mathrm{A}$ in the blood. In diabetic patients, RBP4 levels are correlated with the degree of insulin resistance [2] and with components of the metabolic syndrome, such as BMI, triacylglycerol levels and blood pressure. Conversely, exercise and weight loss have been reported to decrease both RBP4 levels and insulin resistance [2]. However, the link between RBP4 and obesity is controversial, as several other studies have not found RBP4 to be associated with overall obesity or insulin resistance $[3,4]$. In addition, one

H. Sell · J. Eckel $(\bowtie)$

Institute of Clinical Biochemistry and Pathobiochemistry,

German Diabetes Center,

Auf'm Hennekamp 65,

40225 Dusseldorf, Germany

e-mail: eckel@uni-duesseldorf.de

URL: http://www.ddz.uni-duesseldorf.de study reported no difference in circulating RBP4 levels between lean and obese women [5], a finding confirmed very recently [6]. Furthermore, subcutaneous adipose tissue from obese women was characterised by lower RBP4 production compared with lean controls [5]. In these adipose tissue biopsies, GLUT4 levels correlated with RBP4 concentration, making it more likely that RBP4 levels positively correlate with insulin sensitivity in this study. Data on RBP4 production and regulation in human adipocytes are not available, and so the contribution of adipocytes to adipose tissue RBP4 output is difficult to evaluate.

In the present study, primary human preadipocytes were isolated from mammary adipose tissue samples of normal or moderately overweight women (age $40.5 \pm 3.8$ years, BMI $24.2 \pm 2.7 \mathrm{~kg} / \mathrm{m}^{2}, n=4$ in each group) undergoing surgical mammary reduction. All subjects were healthy, free of medication and had no evidence of diabetes according to routine laboratory tests. Adipose tissue samples were dissected from other tissues, pieces of $\sim 10 \mathrm{mg}$ in weight were minced and preadipocytes were isolated by collagenase digestion as previously described [7]. Isolated preadipocytes were seeded in DMEM/Hams F12 medium supplemented with $10 \%$ fetal calf serum and kept in culture overnight. Adipocytes were differentiated over 15 days in a differentiation medium (DMEM/F12, $33 \mu \mathrm{mol} / 1$ biotin, $17 \mu \mathrm{mol} / 1 \mathrm{D}-$ pantothenic acid, $66 \mathrm{nmol} / 1 \mathrm{insulin}, 1 \mathrm{nmol} / 1$ triiodo-L-thyronine, $100 \mathrm{nmol} / \mathrm{l}$ cortisol, $10 \mu \mathrm{g} / \mathrm{ml}$ apotransferrin, $50 \mu \mathrm{g} / \mu \mathrm{l}$ gentamicin, $15 \mathrm{mmol} / \mathrm{l}$ HEPES, $14 \mathrm{mmol} / \mathrm{l} \mathrm{NaHCO}_{3}, \mathrm{pH}$ 7.4). After 15 days of culture, $60-80 \%$ of cells were differentiated as defined by cytoplasm filled with small or large lipid droplets. Differentiated adipocytes were treated with recombinant human adiponectin (Tebu, Offenbach, Germany), troglitazone and recombi- 
nant human TNF- $\alpha$ (Sigma, Taufkirchen, Germany), and then the cells were lysed in $50 \mathrm{mmol} / \mathrm{l}$ HEPES containing $1 \%$ (vol/vol) Triton. Aliquots of $8 \mu \mathrm{g}$ of protein were separated by SDS-PAGE and immunoblotted with antibodies directed against RBP4 (Alexis Biochemicals, Lausen, Switzerland), GLUT4 (Cell Signaling Technology, Frankfurt, Germany), peroxisome proliferator-activated receptor $\gamma$

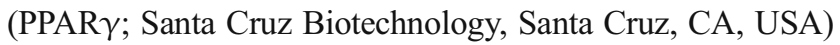
and tubulin (Calbiochem, Merck Biosciences, Schwalbach, Germany). Horseradish peroxidase-conjugated goat antirabbit and goat anti-mouse $\operatorname{IgG}$ antibodies were supplied by Promega (Mannheim, Germany). Statistical analysis was performed using Statview software (SAS, Cary, NC, USA), and a $p$ value of $<0.05$ was considered statistically significant.

Primary human adipocytes produce RBP4 at levels of approximately $5 \mathrm{ng} / \mu \mathrm{g}$ of total cellular protein based on comparison with a recombinant RBP4 standard (data not shown). A very recent publication demonstrates that the RBP4 concentration is very low in isolated human preadipocytes but strongly increases during differentiation into mature adipocytes [6]. Here, we show that production of this adipokine is regulated by TNF- $\alpha$ and the PPAR $\gamma$ agonist troglitazone (Fig. 1a). RBP4 production is not influenced by adiponectin, although adiponectin strongly influences the secretory function of primary adipocytes [7]. Our results show that TNF- $\alpha$ strongly downregulates RBP4 production in adipocytes, a completely unexpected effect, as TNF- $\alpha$-treated adipocytes are insulin resistant [8]. Furthermore, troglitazone increases RBP4 levels by over $100 \%$. This finding is in contrast to observations in mice, where rosiglitazone decreased $R b p 4$ mRNA expression in adipose tissue [1]. It may be speculated that the observed effect of troglitazone in mouse adipose tissue could be due to non-adipocytes. In addition to RBP4, troglitazone significantly stimulated the production of GLUT4 and PPAR $\gamma$ (Fig. 1b,c). When applied overnight, TNF- $\alpha$ did not change GLUT4 production but significantly reduced PPAR $\gamma$ production. GLUT4 downregulation is known to require at least $24 \mathrm{~h}$ of TNF- $\alpha$ treatment, but insulin resistance can be observed as soon as $60 \mathrm{~min}$ after TNF- $\alpha$ administration in human adipocytes [8].

The adipokine RBP4 has recently been shown to link obesity and insulin resistance in rodents [2]. In humans, however, the role of RBP4 is still controversial. In human adipocytes, RBP4 production is downregulated by the proinflammatory cytokine TNF- $\alpha$, which is clearly linked to adipocyte insulin resistance [8]. In a very recent study, secretion of RBP4 and other pro-inflammatory cytokines was downregulated by atrial natriuretic peptide (ANP) [9]. ANP stimulates lipolysis in adipocytes and in humans [10], an effect that can also be attributed to TNF- $\alpha$. It might be that RBP4 production in adipocytes is linked to lipolysis. On the other hand, we found that troglitazone strongly induces
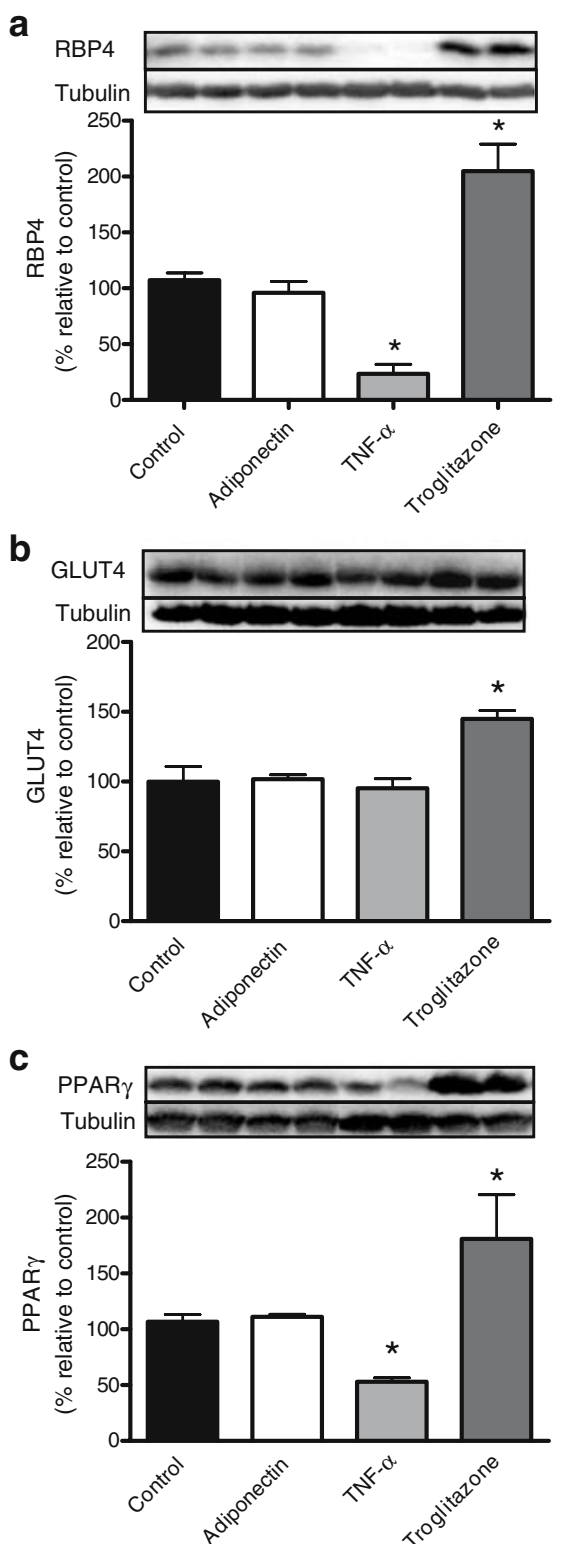

Fig. 1 RBP4 (a), GLUT4 (b) and PPAR $\gamma$ (c) production in human adipocytes treated with $5 \mathrm{nmol} / \mathrm{l}$ adiponectin, $5 \mathrm{mmol} / \mathrm{l}$ troglitazone or $2.5 \mathrm{nmol} / 1 \mathrm{TNF}-\alpha$, as indicated, overnight $(n=3-4) .{ }^{*} p<0.05$ vs control

RBP4 production in human adipocytes, a finding in contrast to data on adipose tissue of mice treated with the PPAR $\gamma$ agonist rosiglitazone [1]. However, the rosiglitazone-induced decreased in RBP4 in mice may reflect improved insulin sensitivity and may not represent the underlying causative effect. Taken together, our data suggest that RBP4 seems to correlate positively with insulin sensitivity and not with insulin resistance in isolated human adipocytes. The present experiments were carried out with adipocytes from mammary fat, which are more similar to subcutaneous adipocytes than to visceral ones. Interestingly, another study working with subcutaneous adipose tissue also revealed controversial data on RBP4 expression, showing that RBP4 mRNA expression 
is decreased in obese women compared with lean controls [5]. Our results point to an important difference in the regulation of RBP4 production between humans and rodents, as well as possible differences between fat depots. The contribution of RBP4 from adipocytes to circulating levels of this adipokine should be evaluated in diabetic and obese patients. The role of RBP4 as a pro-inflammatory adipokine may have to be revised.

Acknowledgements This work was supported by the Ministry of Science and Research of North Rhine-Westphalia, the Federal Ministry of Health, and the Foundation for Pathobiochemistry and Molecular Diagnostics. The secretarial assistance of B. Hurow is gratefully acknowledged.

Duality of interest The authors declare that there is no duality of interest associated with this manuscript.

\section{References}

1. Yang Q, Graham TE, Mody N et al (2005) Serum retinol binding protein 4 contributes to insulin resistance in obesity and type 2 diabetes. Nature 436:356-362

2. Graham TE, Yang Q, Bluher $M$ et al (2006) Retinol-binding protein 4 and insulin resistance in lean, obese, and diabetic subjects. N Engl J Med 354:2552-2563
3. Takashima N, Tomoike H, Iwai N (2006) Retinol-binding protein 4 and insulin resistance. N Engl J Med 355:1392; author reply 1394 1395

4. Stefan N, Hennige AM, Staiger $H$ et al (2007) High circulating retinol-binding protein 4 is associated with elevated liver fat but not with total, subcutaneous, visceral, or intramyocellular fat in humans. Diabetes Care 30:1173-1178

5. Janke J, Engeli S, Boschmann M et al (2006) Retinol-binding protein 4 in human obesity. Diabetes 55:2805-2810

6. Vitkova M, Klimcakova E, Kovacikova M et al (2007) Plasma levels and adipose tissue messenger ribonucleic acid expression of retinol-binding protein 4 are reduced during calorie restriction in obese subjects but are not related to diet-induced changes in insulin sensitivity. J Clin Endocrinol Metab 92:2330-2335

7. Dietze-Schroeder D, Sell H, Uhlig M, Koenen M, Eckel J (2005) Autocrine action of adiponectin on human fat cells prevents the release of insulin resistance-inducing factors. Diabetes 54:20032011

8. Liu LS, Spelleken M, Rohrig K, Hauner H, Eckel J (1998) Tumor necrosis factor-alpha acutely inhibits insulin signaling in human adipocytes: implication of the p80 tumor necrosis factor receptor. Diabetes 47:515-522

9. Moro C, Klimcakova E, Lolmede K et al (2007) Atrial natriuretic peptide inhibits the production of adipokines and cytokines linked to inflammation and insulin resistance in human subcutaneous adipose tissue. Diabetologia 50:1038-1047

10. Lafontan M, Moro C, Sengenes C, Galitzky J, Crampes F, Berlan M (2005) An unsuspected metabolic role for atrial natriuretic peptides: the control of lipolysis, lipid mobilization, and systemic nonesterified fatty acids levels in humans. Arterioscler Thromb Vasc Biol 25:2032-2042 